\title{
The Myelin Vacuolation (mv) Rat with a Null Mutation in the Attractin Gene
}

\author{
Mitsuru Kuwamura, Masaya Maeda, Takashi Kuramoto, Kazuhiro Kitada, \\ Toshiko Kanehara, Mitsuaki Moriyama, Yoshifumi Nakane, Jyoji Yamate, \\ Toshikazu Ushijima, Takao Kotani, and Tadao Serikawa
}

Laboratory of Veterinary Pathology (MK, M. Maeda, T. Kanehara, JY, T. Kotani) and Integrative Physiology (M. Moriyama), Osaka Prefecture University, Osaka, and Institute of Laboratory Animals (YN, KK, TS), Graduate School of Medicine, Kyoto University, Kyoto, and Carcinogenesis Division (T. Kuramoto, TU), National Cancer Center Research Institute, Tokyo, Japan

\begin{abstract}
SUMMARY: We recently found a spontaneous tremor mutant in an outbred colony of Sprague-Dawley rats. The tremor behavior was exhibited from around 3 weeks of age and inherited as an autosomal recessive trait. The mutant rats had variously sized vacuoles in the neuropil and white matter throughout the central nervous system, especially in the brain stem, cerebellum, and spinal cord. Ultrastructurally these vacuoles mainly consisted of splitting of myelin lamella both in the periaxonal and intermyelinic spaces. Linkage analysis using intercross progeny between the myelin vacuolation $(m v)$ rat, named after the pathologic characteristics, and normal control rat strains showed that the $m v$ phenotypes were cosegregated with polymorphic markers adjacent to the Atrn (Attractin, formerly zi [zitter]) locus on rat chromosome 3. A test for allelism suggested that the mv mutation was a new allele in Atrn. In comparison with a marked decrease of $A t r n^{z i} / A r n^{z i}$, Northern blot analysis revealed no expression of Atrn mRNA in the brain of the $m v$ rats. Finally, a genomic deletion including exon 1 of the $m v$ rats was detected by genomic and sequence analyses. Discovery of the rat null mutation $A t r n^{m v}$, different from Atrn ${ }^{z i}$, provides a new animal model for studying the functions of the attractin protein. (Lab Invest 2002, 82:1279-1286).
\end{abstract}

$T$ he human ATRN is a glycoprotein consisting of two isoforms, membrane-type $(200 \mathrm{kDa})$ and secreted-type $(175 \mathrm{kDa})$ as a result of alternative splicing (Duke-Cohan et al, 1998; Gunn et al, 1999; Nagle et al, 1999; Tang et al, 2000). The identification and function of the ATRN have been investigated from immunologic aspects in a secreted form. Initially, ATRN was thought to be a soluble form of the T-cell antigen CD26; however, it has no sequence similarity to CD26. The human ATRN has an enzymatic activity resembling dipeptidyl peptidase IV, a serine type protease with modulatory activity on a number of chemokines, neuropeptides, and peptide hormone. The protein is considered to mediate an interaction between activated $T$ cells and macrophages (DukeCohan et al, 1995, 1998, 2000).

The extracellular region of ATRN contains a CUB domain, three EGF domains, four Plexin domains, and two laminin-type EGF domains. This constitution suggests potential roles in cell adhesion, cell-cell interac-

DOI: 10.1097/01.LAB.0000032375.70196.26

Received February 28, 2002.

This research was supported by Grants-in-Aid for Scientific Research from the Japan Society for the Promotion of Science (13760222 and 12308044). Address reprint requests to: Dr. M. Kuwamura, Laboratory of Veterinary Pathology, Osaka Prefecture University, Sakai, Osaka 599-8531, Japan. E-mail:kuwamura@vet.osakafu-u.ac.jp tions, or signaling (Duke-Cohan et al, 2000; He et al, 2001).

In mice, only membrane-type ATRN has been noticed; mutation in the membrane-type ATRN has been identified in coat color mutant mahogany $(\mathrm{mg})$ mice (Gunn et al, 1999; Lane and Green, 1960; Nagle et al, 1999). The $m g$ mice display a darkened coat color that is agouti dependent (He et al, 2001; Miller et al, 1997). The mechanism by which mahogany modifies agouti has been studied, and it has been indicated that interaction between the ATRN and agouti proteins must be required for signaling through a melanocortin receptor, Mc1r (He et al, 2001). Interestingly, increased basal metabolic rate in mahogany mice suggested involvement of the ATRN, and its function was considered to work though an agouti-independent pathway (Dinulescu et al, 1998; He et al, 2001; Nagle et al, 1999), although suppression of $A^{y}$-induced obesity is specific to an interaction between agouti protein and ATRN.

Mutation in the Atrn has been also identified in the zitter (zi) mutant rat, which is pathologically characterized by tremor behavior, hypomyelination, and vacuolation in the central nervous system (CNS) (Kuramoto et al, 1994, 2001). Furthermore, it has been reported that $m g$ mice also had hypomyelination and vacuolation in the CNS, and a slight body tremor after weaning was confirmed by electromyogram analysis. Transgenic rescue experiments indicated that the membrane-type ATRN plays a critical role in normal 
myelination in the CNS (Kuramoto et al, 2001). The extent of the defects including pathologic findings was dependent on the type of the mutations, Atrn ${ }^{m g-3 J}$, $A_{t r n}{ }^{m g}$, and Atrn ${ }^{m g-L}$ (Gunn et al, 2001). As described above, the ATRN plays multiple roles in regulating physiologic processes such as monocyte-T cell interaction, agouti-related hair pigmentation, energy homeostasis, and myelination.

Recently, the fourth mutation (Atrn ${ }^{m g-65}$ ) in the mouse (Bronson et al, 2001) and the first mutation in the Syrian hamster $\left(A t r{ }^{b t}\right)$ have been reported (Kuramoto et al, 2002). We found a spontaneous mutant rat with tremor behavior in a colony of Sprague-Dawley rats and herein describe the neuropathologic, genetic, and molecular biologic results. This study guided us to the discovery of the null mutation of the Atrn locus in the rat.

\section{Results}

\section{Clinical Behavior}

The homozygous rats ( $m v / m v$ rats) started to show tremor behavior around 3 weeks of age, whereas the heterozygous rats exhibited no behavioral anomaly. The tremor was revealed more clearly when external stimuli such as auditory or touch were given and when the rats started to move. Hind body trembling was observed after 4 weeks of age when walking. After 2 months of age, some rats occasionally showed syncopal convulsion to the external stimuli with duration of about 1 minute. These signs progressed with advancing age, and most rats survived at least to 25 weeks of age.

\section{Neuropathology}

In $m v$ rats $(m v / m v)$, variously sized vacuoles were observed in the neuropil and white matter throughout the CNS; in particular, prominent vacuolization was found in the thalamus, midbrain (Fig. 1A), cerebellar nuclei, medulla oblongata, and spinal cord. The vacuoles were also observed in the cerebral cortex, putamen, hippocampus, and cerebellum (Fig. 1B) to a lesser extent. At 2 and 3 weeks of age, vacuoles were mainly observed in the brain stem. Severity and extent of anatomical distribution of vacuolization progressed with advancing age. Large vacuoles found in 10- and 25-week-old $m v$ rats often contained eosinophilic granular materials, so-called "spheroids" (Fig. 1C).

The Luxol fast blue staining pattern was weaker in the vacuolated areas of $m v$ rats as compared with that in control rats. Myelin basic protein (MBP) immunohistochemistry clearly demonstrated weakened reactivity in the areas with vacuolization (Fig. 2). Immunohistochemistry for glial fibrillary acidic protein revealed enhanced reactivity in the vacuolated areas, indicating the presence of many hypertrophic astrocytes (Fig. 3).

Ultrastructurally, these vacuoles were present in the periaxonal and intermyelinic spaces, consisting mainly of splitting of myelin lamella (Fig. 4A). Occasionally, vacuolated myelin contained a dystrophic axon with aggregation of dense bodies and granulated materials (Fig. 4B). Aberrant myelination, consisting of a bundle of myelin lamella surrounding two or three axons, was found in the white matter of $m v$ rats (Fig. 4C).

At 4 weeks of age, the density of myelinated fibers in the thoracic spinal cord was apparently decreased in $m v$ rats in comparison with that of controls (Fig. 5, A and $\mathrm{B}$ ). Myelin thickness in $m v$ rats was significantly lower than in controls (Fig. 5C), whereas mean axonal area, perimeter, and diameter were significantly greater in $m v$ rats than in controls (Fig. 5C). These results indicated that $m v$ rats had thin myelin and relatively thick axons (Table 1). No apparent abnormality was found in the dorsal and lateral roots and in the sciatic nerves.

\section{Genetic Analysis}

Preliminary linkage analysis was conducted with 13 backcross progenies from $(\mathrm{F} 344 \times m v /+) \mathrm{F}_{1}(m v /+) \times$ $\mathrm{mv} /+$ using 54 simple sequence length polymorphism (SSLP) markers randomly selected from chromosomes 1 to 20. The mutant phenotypes were cosegregated exclusively with the markers (D3Rat20, D3Rat219, D3Rat258, D3M2Mit257) adjacent to the zi (Atrn) locus on rat chromosome 3 , which suggested a

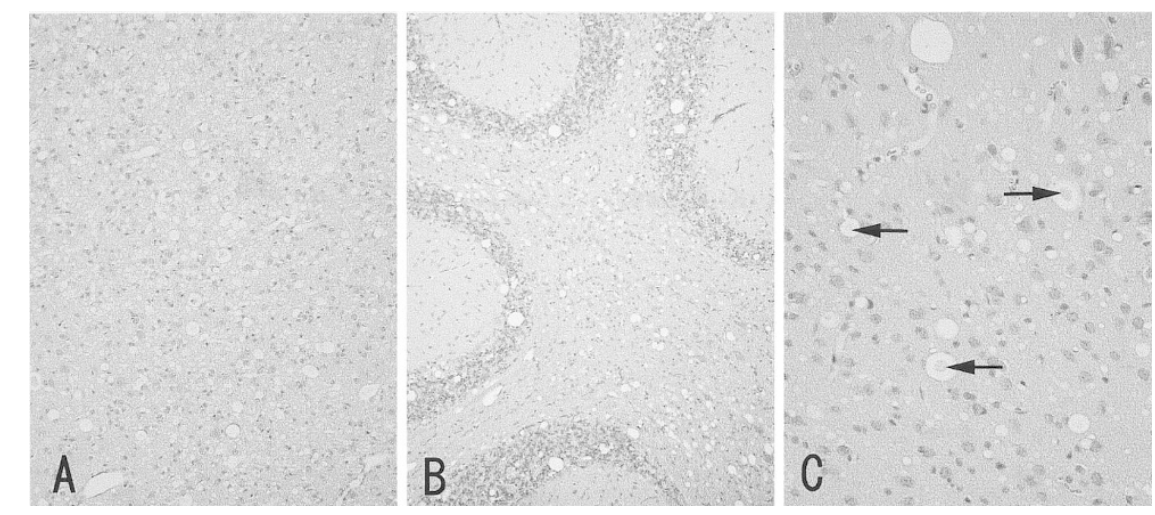

Figure 1.

Vacuolization in the central nervous system of the $m v$ rat. Variously sized vacuoles were observed in the midbrain (A), cerebellum (B), and cerebral cortex (C). Note vacuoles containing eosinophilic granular materials (C, arrows); hematoxylin and eosin. Original magnifications: $A$ and $B, \times 100 ; C, \times 200$. 


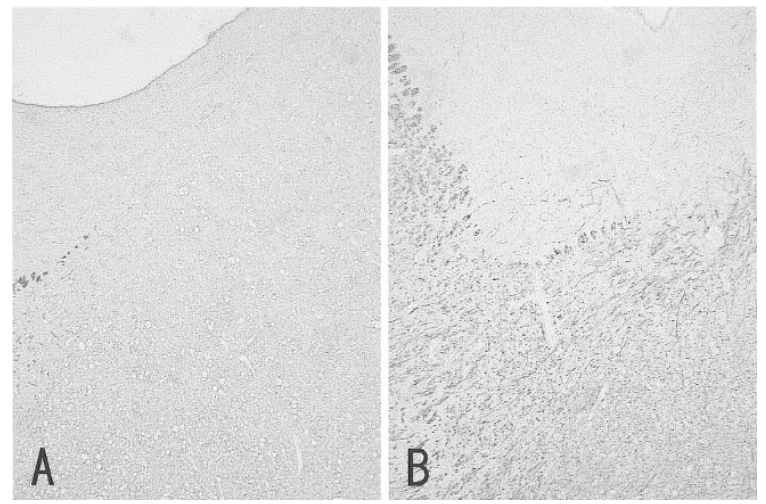

Figure 2.

Immunohistochemistry for myelin basic protein (MBP). MBP reactivity was weakened in the vacuolated area in a $m v / m v$ rat. A, $m v / m v$ rat. B, control rat. Midbrain. Original magnification: $\times 100$.

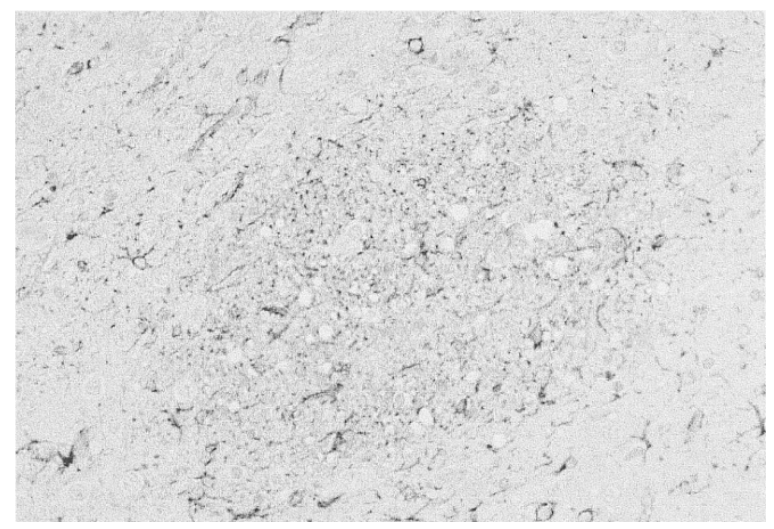

Figure 3.

Immunohistochemistry for glial fibrillary acidic protein (GFAP). Many hypertrophic astrocytes were found in the vacuolated area. Thalamus. Original magnification: $\times 200$

close relationship between $m v$ and zi mutations (data not shown).

Further analysis was conducted with 92 intercross progeny using SSLP markers adjacent to the Atrn locus. Additional haplotype analysis in the $\mathrm{mv} / \mathrm{mv}$ homozygotes revealed that the $m v$ locus is mapped between the D3Mit13 and D3M2Mit257 and there is no recombination with the Pdyn marker (Fig. 6). To confirm the relationship between the $m v$ and Atrn loci, we crossed male $\mathrm{mv} /+$ rats, whose genotypes were proven from previous breeding record, with $A t r n^{z i}$ / $A_{\text {trn }}^{+}$rats. Of 16 offspring, 2 rats (12.5\%) showed the indistinguishable tremor behavior and pathologic changes in the CNS, as found in $\mathrm{mv} / \mathrm{mv}$ or Atrn ${ }^{2 i} /$ Atrn $^{2 i}$ rats. In the intercross progeny prepared to examine the effects of $m v$ mutation in pigmentation, rats with a more darkened coat color in the dorsal area, so-called mahogany color, were obtained (Fig. 7).

\section{No Expression of Atrn in mv/mv Rats}

Expression of the Atrn gene was analyzed in the brain of the affected rats $(\mathrm{mv} / \mathrm{mv})$ and normal control rats by Northern blot hybridization (Fig. 8). When we used a probe corresponding to exons 7 to 9 that would detect both membrane- and secreted-type Atrn transcript, neither transcript was observed in the affected rats.

\section{Mutation of Atrn in mv Rats}

Because no Atrn transcripts were detected in $\mathrm{mv} / \mathrm{mv}$ rats, it was predicted that $m v$ rats carried a mutation involving exon 1 of Atrn. Nucleotide sequences of a 5'-noncoding region spanning $3754 \mathrm{bp}$, an intronic sequence after exon 1 spanning $2567 \mathrm{bp}$, and an intronic sequence preceding exon 2 spanning $2347 \mathrm{bp}$ were determined (GenBank accession numbers AB069686, AB069687). The length of intron 1 was not determined because it was too long to be amplified by PCR. A DNA fragment derived from intron 1 (Fig. 9A) did not detect any bands in Southern blot analysis of $\mathrm{mv} / \mathrm{mv}$ rats (Fig. 9B). To estimate the lacking genomic region, we developed four sequence tagged site markers and conducted sequence tagged site-content mapping using the wild-type and $\mathrm{mv} / \mathrm{mv}$ genomic DNAs as templates. One of the breakpoints for the deletion in the genome of $\mathrm{mv} / \mathrm{mv}$ rats was located between positions -719 ( 3 ' end of primer "a" in Fig. 9A) and -632 (5' end of primer "b" in Fig. 9A) (Fig. 9C). Other breakpoints could not be determined because the entire sequence of intron 1 could not be obtained. However, it was revealed that an $\sim 3.6-k b$ genomic region that included the 630 -bp 5'-noncoding region and the entire exon 1 was deleted in $m v$ rats.

\section{Discussion}

We found a mutant rat with tremor behavior that is pathologically characterized by vacuole formation in the CNS, consisting mainly of splitting of the myelin lamella. This mutant rat was designated as $m v$ (myelin vacuolation) because of its pathologic characteristics. Clinical signs and pathologic findings in the $m v$ rats were very similar to those seen in the $A t r n^{z i} / A t r n^{z i}$ (zitter) rats. The zitter rats revealed a marked decrease in Atrn mRNA in the brain, and an 8-bp deletion at a splice donor site of Atrn was discovered, which was expected to result in aberrant and unstable transcripts (Kuramoto et al, 2001). On the other hand, no expressions of either isoform of the Atrn transcripts was found in the $\mathrm{mv} / \mathrm{mv}$ rats, and an $\sim 3.6-\mathrm{kb}$ genomic region that included the 630-bp 5'-upstream region and the entire exon 1 was deleted in the rats.

Northern blot analysis of $\mathrm{mg}$ mice revealed the Atrn ${ }^{m g-3 J}$ mutant has no detectable Atrn transcript, whereas $A t r n^{m g}$ and $A t r n^{m g-L}$ mutants have a small amount of normal sized transcript and more abundant aberrant isoforms (Gunn et al, 1999; Nagle et al, 1999). Compared with the severity of vacuolization in the CNS among three different Atrn mutant alleles, it has been reported that $A t r n^{m g-3 J}$ homozygotes showed most the severe lesions and $A t r n^{m g-L}$ homozygotes the least (Gunn et al, 2001). This observation suggested that the severity of vacuolization correlates with the expressed levels of Atrn mRNA and indicated 


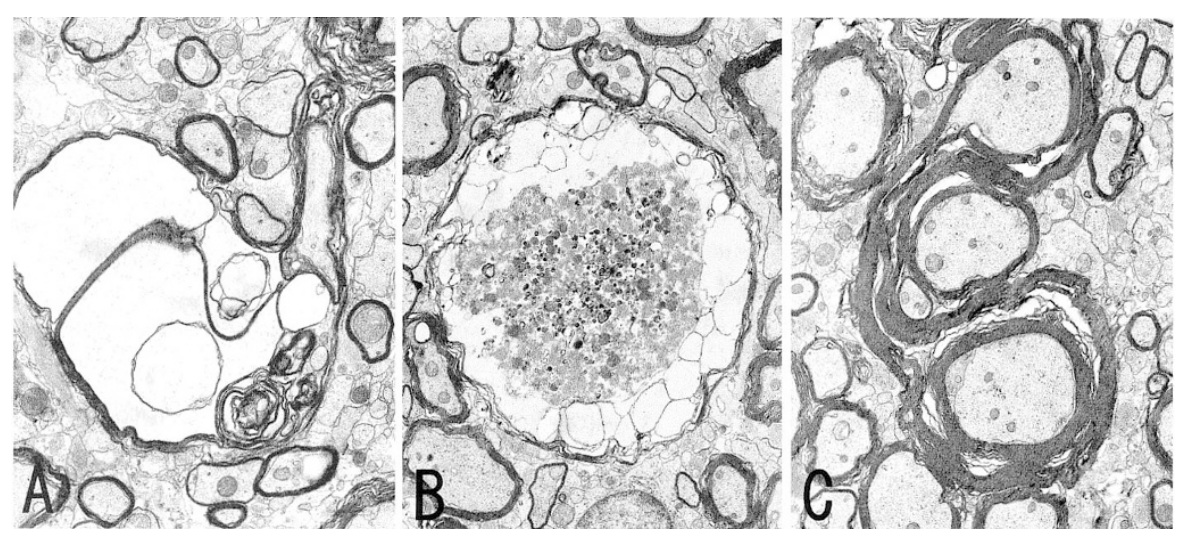

Figure 4.

Electron micrographs of the $m v$ rat. A, Vacuoles consisting of splitting of myelin lamella. B, Myelin vacuolation containing a dystrophic axon with aggregation of the dense bodies and granulated materials. $\mathrm{C}$, Aberrant myelination consisting of a bundle of myelin lamella surrounding multiple axons. Original magnifications: A, $\times$ 5000; B and C, $\times 7500$.
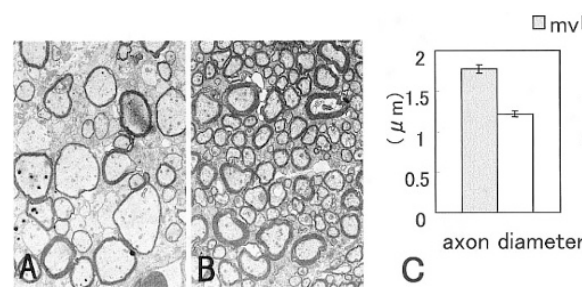

$\square \mathrm{mv} \square$ control

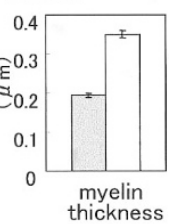

Figure 5.

Morphometric analysis in cross-sectioned ventral areas of thoracic spinal cord in $m v(A)$ and control (B) rats. Density of myelinated fibers was decreased in the $m v$ rat. C, Myelin thickness in $m v$ rat was significantly lower than in controls, whereas axonal diameter in $m v$ rat was greater than in controls. Original magnifications: $\mathrm{A}$ and $\mathrm{B}, \times 2500$.

Table 1. Mean Values of Myelinated Fibers Measured in the Anterior Funiculus of the Thoracic Spinal Cords in $m v$ and Control Rats

\begin{tabular}{|c|c|c|}
\hline & $m v(n=3)$ & control $(n=3)$ \\
\hline Axonal area $\left(\mu \mathrm{m}^{2}\right)^{a}$ & $3.107 \pm 0.203$ & $1.450 \pm 0.096$ \\
\hline Axonal perimeter $(\mu \mathrm{m})^{a}$ & $6.043 \pm 0.185$ & $4.280 \pm 0.138$ \\
\hline Axonal diameter $(\mu \mathrm{m})^{a}$ & $1.773 \pm 0.052$ & $1.215 \pm 0.035$ \\
\hline Fiber area $\left(\mu \mathrm{m}^{2}\right)^{b}$ & $4.500 \pm 0.269$ & $3.497 \pm 0.212$ \\
\hline Fiber perimeter $(\mu \mathrm{m})^{c}$ & $7.303 \pm 0.210$ & $6.601 \pm 0.196$ \\
\hline Fiber diameter $(\mu \mathrm{m})^{b}$ & $2.162 \pm 0.060$ & $1.915 \pm 0.052$ \\
\hline Myelin thickness $(\mu \mathrm{m})^{d}$ & $0.194 \pm 0.005$ & $0.350 \pm 0.009$ \\
\hline$g$-ratio ${ }^{a}$ & $0.810 \pm 0.003$ & $0.626 \pm 0.004$ \\
\hline Myelin ratio ${ }^{d}$ & $0.190 \pm 0.003$ & $0.374 \pm 0.004$ \\
\hline
\end{tabular}

All values are expressed as mean \pm SE of three rats.

${ }^{a} m v$ is significantly greater than controls $(p<0.0001)$.

${ }^{b} \mathrm{mv}$ is significantly greater than controls $(p<0.005)$.

${ }^{c} m v$ is significantly greater than controls $(p<0.05)$.

${ }^{d} \mathrm{mv}$ is significantly smaller than controls $(p<0.0001)$.

crucial roles of Atrn for spongy-form degeneration in the CNS.

Atrn mRNA was found widely distributed throughout the CNS; a very high level of expression was observed in the olfactory system, some limbic structures, brain stem, cerebellum, and spinal cord in rats (Lu et al, 1999). Severe vacuolization was found in the brain stem, cerebellum, and spinal cord, whereas less mild lesions were observed in the olfactory system and hippocampus in both the rat mutants $A_{t r n}{ }^{m v} / A^{2} r^{m v}$ and Atrn $^{z i} /$ Atrn $^{z i}$ (Kondo et al, 1991a, 1991b, 1992). Although the Atrn mRNA expression pattern and the severity of vacuolization are roughly matched, some differences existed in particular regions of the CNS. Thus, in animals with dysfunction of ATRN, the precise mechanism resulting in the vacuolation in the CNS remains to be elucidated.

Besides hypomyelination and aberrant myelination, $A t r n^{z i} / A_{t r n}{ }^{z i}$ rats have abnormal membranous structures associated with oligodendroglial nuclear membrane and aberrant myelin sheaths in the CNS and peripheral nerves (Kondo et al, 1993, 1995). Such membranous abnormalities were also found in mitochondria, in cisternae of rough endoplasmic reticulum, and in lysosomes. These findings in Atrn ${ }^{2 i} / A t r n^{2 i}$ rats suggested that functional abnormalities in membrane biosynthesis, including the myelin sheaths and nuclear membranes in oligodendrocytes, were closely related to the hypomyelination. Therefore, it remains to be elucidated whether dysfunction in ATRN primarily targets the axons, myelin, or membrane biosynthesis.

Recently, a new mice mutant in Atrn allele, $A_{t r n}{ }^{\mathrm{mg}-6 J}$, was found in a stock of Mus castaneus mice and showed sprawling gait, tremors, and severe vacuolization in the CNS in addition to the mahogany coat color (Bronson et al, 2001). Vacuoles in the Atrn ${ }^{m v}$ / $A t r n^{m v}$ rats were exclusively found in the myelin lamella as seen in $A t r n^{z i} / A t r n^{z i}$ rats, whereas those in the $A_{t r n}{ }^{\mathrm{mg}-6 \mathrm{~J}} / A_{t r n}{ }^{\mathrm{mg}-6 \mathrm{~J}}$ mice were identified in axons, dendrites, and neuronal soma. As for the extent of defects in the two mutations, it is recommended to compare the effects of the mutant alleles with the same genetic background, as examined in three congenic mice, C3H/HeJ-Atrn ${ }^{m g}, A_{t r n}{ }^{m g-L}$, and Atrn ${ }^{m g-3 J}$ (Gunn et al, 2001). Certainly, the sprawling of CAST/ Ei-Atrn ${ }^{m g-6 J} / A_{t r n}{ }^{m g-6 J}$ was not observed in $\mathrm{C} 3 \mathrm{H} / \mathrm{SnJ}-$ Atrn $^{m g-6 J} /$ Atrn $^{m g-6 J}$ (Bronson et al, 2001), and the extent of mutant phenotypes was more prominent when we developed congenic rat strains WTC-Atrn ${ }^{2 i}$ / $A t r n^{z i}$ from the original zitter rat (Kuramoto et al, 1998). 


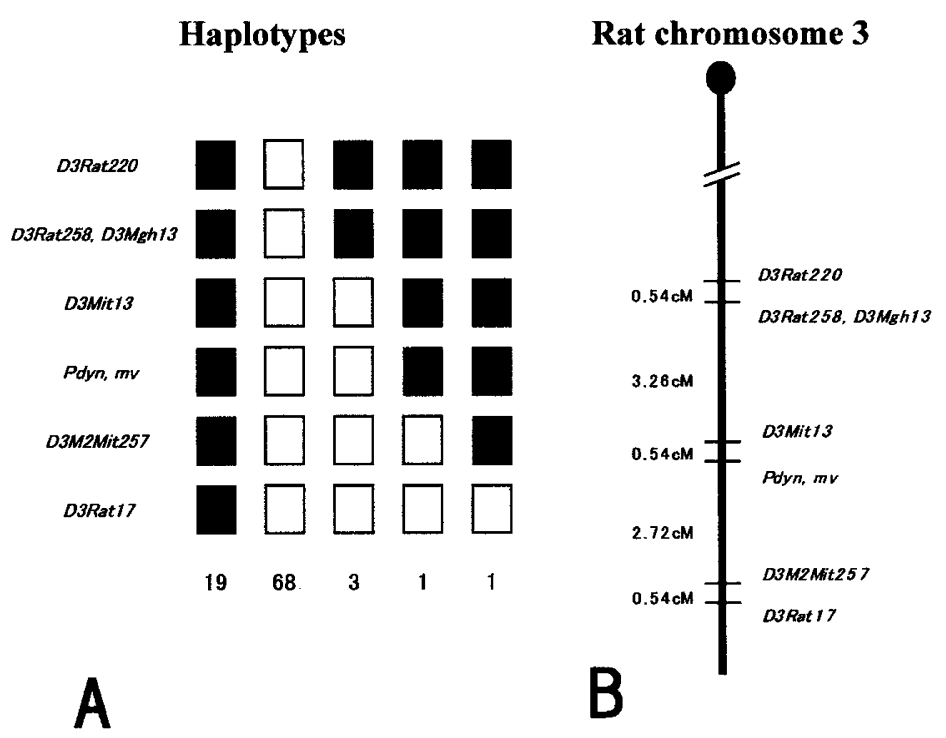

Figure 6.

Mapping of the mv mutation. A, Distribution of haplotypes for rat chromosome 3 ( $\mathrm{Chr} 3$ ) observed among the 92 intercross progeny. Black boxes indicate homozygous for $m v$ allele. White boxes represent heterozygous for $m v$ allele or wild-type rats. B, Genetic linkage map of Chr 3 including the $m v$ locus. Distances between loci are shown to the left, calculated with the Map Manager program.

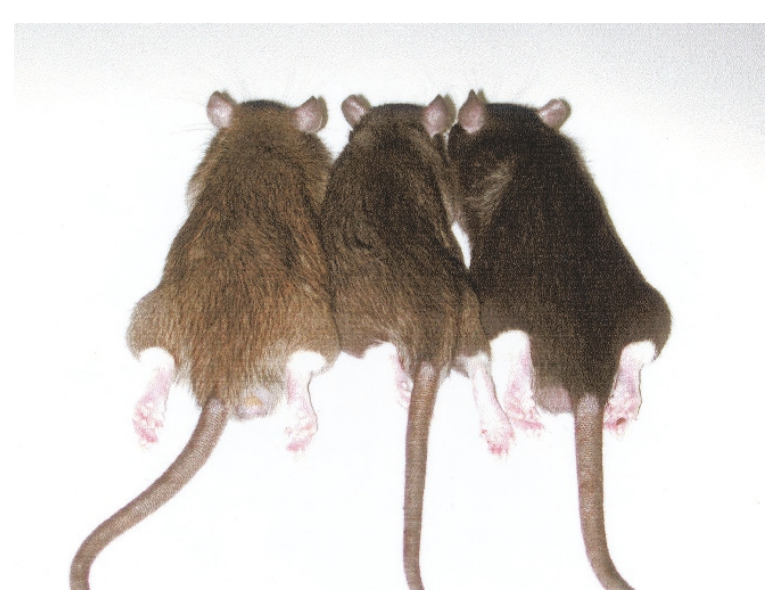

Figure 7.

Color coat effect of $m v$ mutation. $m v / m v$ rat (center) showed more darkened coat color than agouti rat without $m v$ allele (left). The right rat was nonagouti control.

Therefore, the syncopal convulsion observed in the original Atrn $^{m v} /$ Atrn $^{m v}$ rats has to be reevaluated under a different background.

$\mathrm{Mg}$ mice were originally recognized as a modifier of agouti coat color; homozygotes for Atrn ${ }^{m g}$ produce mostly eumelanin, although nonmutant mice have hairs with a subapical band of pheomelanin on an eumelanic background (Lane and Green, 1960; Miller et al, 1997). We also demonstrated a darker coat color in nonalbino agouti rats homozygous for the Atrn ${ }^{m v}$ allele than in agouti rats without the mutant allele, as shown in the Atrn ${ }^{z i}$ mutation (Kuramoto et al, 2001).

In conclusion, a novel mv mutant rat is characterized by tremor behaviors and has severe vacuolation in the CNS. The present study demonstrates defective expression of Atrn mRNA and a possible large deletion in

\section{Rat chromosome 3}




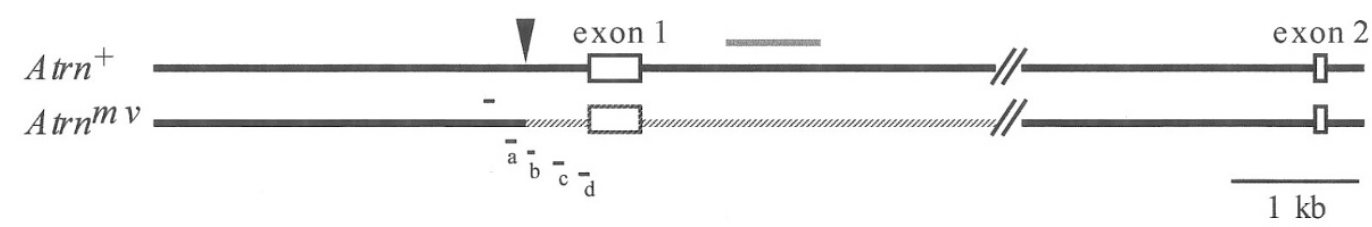

$\mathrm{B}$
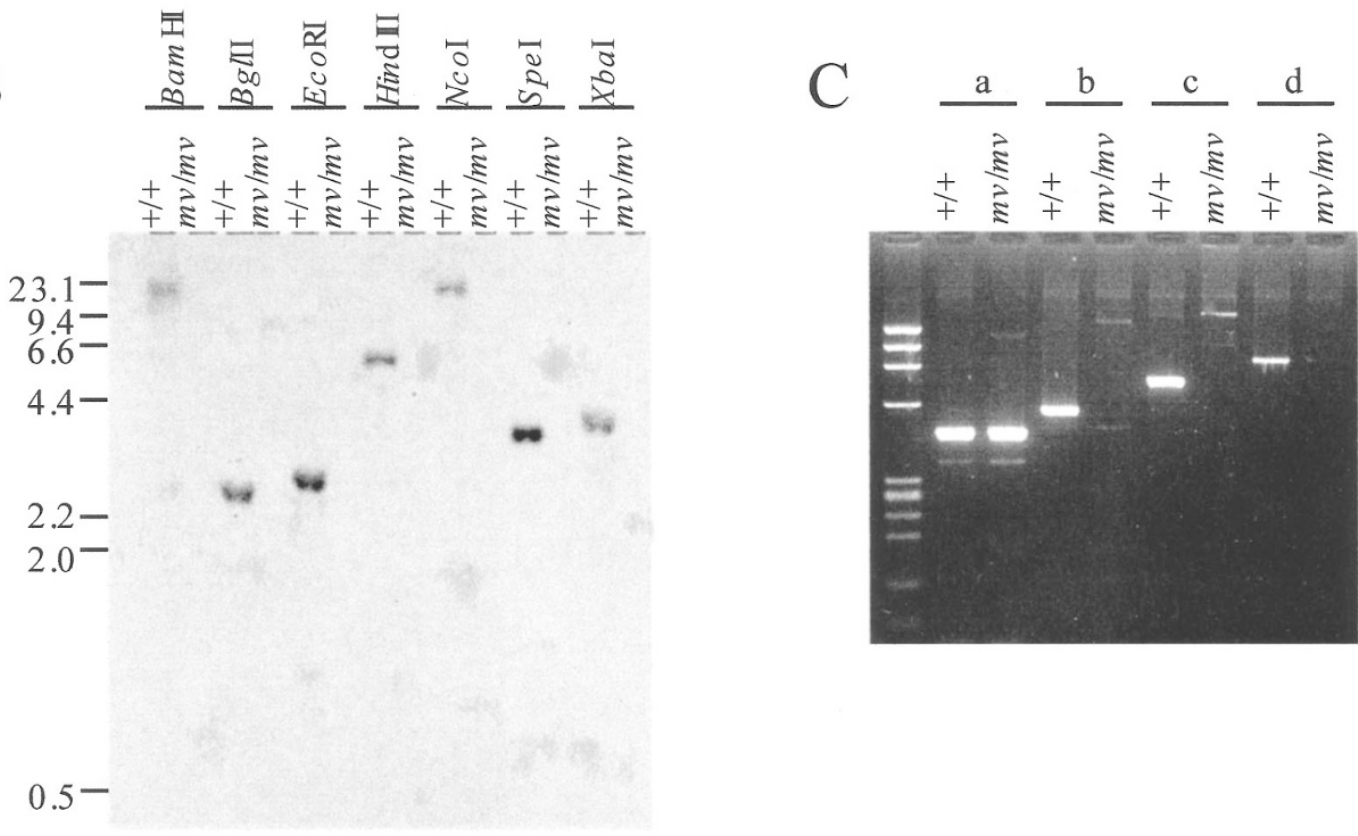

\section{Figure 9.}

Mv mutation at rat Atrn gene. A, Schematic representation of the Atrn gene around the $m v$ deletion at exon 1. Nucleotide sequences of the wild-type allele (upper) are deposited in GenBank with accession numbers AB069686 and AB069687. The length of intron 1 could not be determined. The region of the mv deletion is indicated by a dotted line. The $5^{\prime}$ end of the deletion is indicated by an arrowhead. B. Southern blot hybridization probed with the fragment derived from the $m v$ deletion region. The fragment used as a hybridization probe is indicated by the gray line in A. No hybridization signals were observed in $m v / m v$ rats. C, Determination of 5 '-breakpoint of the $m v$ deletion. The positions of the primers are indicated in A. PCRs were performed with the common oligonucleotide (TCAAAGTCCAGCCCAGTACA) as the sense primer and four oligonucleotides (a, GGAGCACTCTCAGCCTTACA; b, GCAGGCCAGGTCTATGTGTA; c, GTCCAAAAAGCTGCCTAATG; d, CCCAGCGAACACATACACAC) as the antisense primers. Molecular size markers are $\phi X 174$-Haelll. Primer a generated a PCR product of the expected size from $m v / m v$ rats, whereas primers $b$, $c$, and d generated no PCR products from $\mathrm{mv} / \mathrm{mv}$ rats.

\section{Histopathology and Immunohistochemistry}

Rats were killed under ether anesthesia at 1, 2, 3, 4, 10 , and 25 weeks of age. At least four affected mutant and control rats each were examined at each point. Brains and spinal cords were removed and fixed with $10 \%$ neutral buffered formalin and embedded in paraffin. Paraffin sections were cut at $4 \mu \mathrm{m}$ and stained with hematoxylin and eosin and Luxol fast blue stain for myelin.

For the detection of MBP and glial fibrillary acidic protein (GFAP), immunohistochemistry was applied to the paraffin sections of brains and spinal cords with an avidin-biotin peroxidase kit (Vectastain, Burlingame, California). Primary antibodies used were rabbit polyclonal antibodies against GFAP (Dako, Glostrup, Denmark; 1:1000) and MBP (Dako; prediluted). 3,3'diaminobenzidine tetrahydrochloride (Dojindo, Osaka, Japan) was used for visualization.

\section{Electron Microscopy and Morphometric Analysis}

Perfusion fixation through the left ventricle was performed with $4 \%$ paraformaldehyde in $0.1 \mathrm{~m}$ phosphate buffer. Removed brains and spinal cords were stored in $2 \%$ paraformaldehyde and $2.5 \%$ glutaraldehyde in $0.1 \mathrm{M}$ PB and postfixed with $2 \%$ osmic acid for 2 hours and embedded in Epoxy resin. Ultrathin sections were double stained with uranyl acetate and lead citrate and examined in a Hitachi $\mathrm{H}-7500$ electron microscopy (Hitachi, Tokyo, Japan).

Morphometric analysis was performed on the myelinated fibers from the anterior funiculus of the thoracic spinal cord. Several fields in the transverse sections were photographed at a magnification of $\times 2500$. Photo figures were digitized, and 100 myelinated fibers were analyzed by computer-aided morphometric system (Mac Scope; Mitani Corporation, Fukui, Japan). Axonal areas and perimeters were 
measured along the inner border of the myelin sheath, and nerve fiber areas and perimeters were measured along the outer border. Axonal and nerve fiber diameters and myelin thickness were calculated from the obtained data.

\section{Genetic Analysis}

We mated a male heterozygous for the mv mutation $(m v /+)$ with several females of the F344/Crj strain. Subsequently, $F_{1} m v /+$ female rats were backcrossed with their $\mathrm{mv} /+$ father; $13 \mathrm{mv} / \mathrm{mv}$ progeny for preliminary linkage analysis were obtained. Furthermore, we mated a male homozygous $m v(\mathrm{mv} / \mathrm{mv})$ and females of the $\mathrm{ACl} / \mathrm{N}$ strain, then intercrossed the $\mathrm{F}_{1}$ rats and produced 92 offspring for detailed mapping. Genomic DNA was extracted from the spleen of the animals, and 50 SSLP markers, evenly distributed over the whole rat genome, were used for the analysis of haplotype segregations. PCR procedures were performed with 35 cycles (denaturation at $94^{\circ} \mathrm{C}$ for 30 seconds, annealing at $55^{\circ} \mathrm{C}$ for 60 seconds, extension at $72^{\circ} \mathrm{C}$ for 45 seconds). Detailed methods for PCR amplification for SSLP markers were described previously (Serikawa et al, 1992). PCR products were examined on $4 \%$ NuSieve agarose gels with ethidium bromide staining.

For the allelism test, matings between the proven $m v$ heterozygous $(m v /+)$ and $z i$ heterozygous $(z i /+)$ were conducted. Clinical behavior and histopathology in the CNS were checked in the $F_{1}$ rats.

To test the effect of the mv mutation on coat color, $(\mathrm{F} 344 \times \mathrm{SD}-m v /+) \mathrm{F}_{1}(\mathrm{a} / \mathrm{a}, \mathrm{mv} /+$, albino) were crossed to $A C l / N(A / A,+/+)$ to introduce the $m v$ allele into a nonalbino, agouti background; then intercross matings were conducted. The genotypes at the agouti locus were checked by a SSLP marker in intron 2 of rat agouti (5'-GGG AAA GAC CAT AGC TGC TA-3' and 5'-GGA GTT GGC AAC TGA AGA AC-3'). The genotypes at the $m v$ locus were determined by both clinical behavior and SSLP markers cosegregated to the $m v$ allele.

\section{RNA Isolation and Northern Blotting}

Total RNA was isolated from the brain of a 12-weekold rat by using ISOGEN (NIPPON GENE, Tokyo, Japan). Poly-A RNA was purified by using OligotexdT30 Super (Roche Diagnostics, Mannheim, Germany). Two micrograms of poly- $A^{+}$RNA was electrophoresed on formaldehyde-denaturing agarose gel and blotted onto Hybond $\mathrm{N}+$ nylon membrane (Amersham Pharmacia Biotech, Buckinghamshire, England). The hybridization probe corresponded to exon 7 to 9 and was expected to detect both membrane- and secreted-type Atrn transcripts (Kuramoto et al, 2001).

\section{Southern Blot Hybridization}

Genomic DNA was extracted from the liver, and Southern blotting was performed according to the standard protocol (Sambrook and Russell, 2001). The filters were hybridized with the probe that corre- sponded to the region between $464 \mathrm{bp}$ and $1176 \mathrm{bp}$ from the donor site of intron 1.

\section{Determination of Nucleotide Sequence Around Exon 1}

From a PAC clone, 112F10, which spanned the entire Atrn gene (Kuramoto et al, 2001), subclones that contained exon 1 or exon 2 were isolated. The subclones were directly sequenced using primers specific for the Atrn gene.

\section{Statistics}

For the morphometric analysis, the mean values were compared between $m v$ and control rats using Student's grouped $t$ test. Statistical significance was accepted at the $5 \%$ level.

\section{References}

Bronson RT, Donahue LR, Samples R, Kim JH, and Naggert JK (2001). Mice with mutations in the mahogany gene Atrn have cerebral spongiform changes. J Neuropathol Exp Neurol 60:724-730.

Dinulescu DM, Fan W, Boston BA, McCall K, Lamoreux ML, Moore KJ, Montagno J, and Cone RD (1998). Mahogany (mg) stimulates feeding and increases basal metabolic rate independent of its suppression of agouti. Proc Natl Acad Sci USA 95:12707-12712.

Duke-Cohan JS, Gu J, McLaughlin DF, Xu Y, Freeman GJ, and Schlossman SF (1998). Attractin (DPPT-L), a member of the CUB family of cell adhesion and guidance proteins, is secreted by activated human $T$ lymphocytes and modulates immune cell interactions. Proc Natl Acad Sci USA 95:1133611341.

Duke-Cohan JS, Morimoto C, Rocker JA, and Schlossman SF (1995). A novel form of dipeptidyl peptidase IV found in human serum: Isolation, characterization and comparison with T lymphocyte membrane dipeptidylpeptidase IV (CD26). J Biol Chem 270:14107-14114.

Duke-Cohan JS, Tang W, and Schlossman SF (2000). Attractin: A cub-family protease involved in T cell-monocyte/ macrophage interactions. Adv Exp Med Biol 477:173-185.

Gunn TM, Inui T, Kitada K, Ito S, Wakamatsu K, He L, Bouley DM, Serikawa T, and Barsh GS (2001). Molecular and phenotypic analysis of attractin mutant mice. Genetics 158: 1683-1695.

Gunn TM, Miller KA, He L, Hyman RW, Davis RW, Azarani A, Schlossman SF, Duke-Cohan JS, and Barsh GS (1999). The mouse mahogany locus encodes a transmembrane form of human attractin. Nature 398:152-156.

He L, Gunn TM, Bouley DM, Lu XY, Watson SJ, Schlossmann SF, Duck-Cohan JS, and Barsh GS (2001). A biochemical function for attractin in agouti-induced pigmentation and obesity. Nat Genet 27:40-47.

Kondo A, Nagara H, Akazawa K, Tateishi J, Serikawa T, and Yamada J (1991a). CNS pathology in the neurological mutant rats zitter, tremor and zitter-tremor double mutant (spontaneously epileptic rat, SER). Brain 114:979-999.

Kondo A, Sato Y, and Nagara H (1991b). An ultrastructural study of oligodendrocytes in zitter rat: A new animal model for hypomyelination in the CNS. J Neurocytol 20:929-939. 
Kondo A, Sendoh S, Akazawa K, Sato Y, and Nagara H (1992). Early myelination in zitter rat: Morphological, immunocytochemical and morphometric studies. Dev Brain Res 67:217-228.

Kondo A, Sendoh S, Miyata K, and Takamatsu J (1995). Spongy degeneration in the zitter rat: Ultrastructural and immunohistochemical studies. J Neurocytol 24:553-544.

Kondo A, Sendoh S, Takamatsu J, and Nagara H (1993). The zitter rat: Membranous abnormality in the Schwann cells of myelinated nerve fibers. Brain Res 613:173-179.

Kuramoto T, Kitada K, Inui T, Sasaki Y, Ito K, Hase T, Kawagachi S, Ogawa Y, Nakao K, Barsh GS, Nagao M, Ushijima T, and Serikawa T (2001). Attractin/Mahogany/Zitter plays a critical role in myelination of the central nervous system. Proc Natl Acad Sci USA 98:559-564.

Kuramoto T, Mori M, Yamada J, and Serikawa T (1994). Tremor and zitter, causative mutant genes for epilepsy with spongiform encephalopathy in spontaneously epileptic rat (SER), are tightly linked to synaptobrevin 2 and prion protein genes, respectively. Biochem Biophys Res Commun 200: 1161-1168.

Kuramoto T, Nomoto T, Fujiwara A, Mizutani M, Sugimura T, and Ushijima T (2002). Insertional mutation of the Attractin gene in the black tremor hamster. Mamm Genome 13:36-40.

Kuramoto T, Yamasaki K, Kondo A, Nakajima K, Yamada M, and Serikawa T (1998). Production of WTC.Zi-zi rat congenic strain and its pathological and genetic analyses. Exp Anim $47: 75-81$.
Lane PW and Green MC (1960). Mahogany, a recessive color mutation in linkage group $V$ of the mouse. J Hered 51:228230.

Lu XY, Gunn TM, Shieh KR, Barsh GS, Akil H, and Watson SJ (1999). Distribution of mahogany/attractin mRNA in the rat central nervous system. FEBS Lett 462:101-107.

Miller KA, Gunn TM, Carrasquillo MM, Lamoreux ML, Galbraith DB, and Barsh GS (1997). Genetic studies of the mouse mutations mahogany and mahoganoid. Genetics 146: 1407-1415.

Nagle DL, McGrail SH, Vitale J, Woolf EA, Dussault BJ Jr, DiRocco L, Holmgren L, Montagno J, Bork P, Huszar D, Fairchild-Huntress V, Ge P, Keilty J, Ebeling C, Baldini L, Gilchrist J, Burn P, Carlson GA, and Moore KJ (1999). The mahogany protein is a receptor involved in suppression of obesity. Nature 398:148-152.

Sambrook J and Russell DW (2001). Molecular cloning: A laboratory manual, 3rd ed. Cold Spring Harbor, NY: Cold Spring Harbor Laboratory Press.

Serikawa T, Kuramoto T, Hilbert P, Mori M, Yamada J, Dubay CJ, Lindpaintner K, Ganten D, Guénet JL, Lathrop GM, and Beckmann JS (1992). Rat gene mapping using PCR-analyzed microsatellites. Genetics 131:701-721.

Tang W, Gunn TM, McLaughlin DF, Barsh GS, Schlossman SF, and Duke-Cohan JS (2000). Secreted and membrane attractin result from alternative splicing of the human ATRN gene. Proc Natl Acad Sci USA 97:6025-6030. 